\title{
IMPLICATIONS OF HADAMARD'S CONDITIONS FOR ELASTIC STABILITY WITH RESPEGT TO UNIQUENESS THEOREMS
}

\author{
J. L. ERICKSEN AND R. A. TOUPIN
}

Introduction. The purpose of this paper is to discuss implications of Hadamard's condition for elastic stability $(\mathbf{2}, \S 269)$ with respect to uniqueness of solutions of boundary value problems in the theory of small deformations superimposed on large. We show that a slightly refined form of his condition implies a uniqueness theorem for displacement boundary value problems. We construct a counter-example showing that his condition does not imply uniqueness of solutions for one type of stress boundary value problem. Hadamard (2, Ch. VI) showed that his condition implies the reality of all possible velocities of propagation of acceleration waves. To our knowledge, this is the only other known consequence of his condition.

Truesdell (8) has focused attention on the question of what conditions should be imposed on the strain energy to exclude physically unacceptable behavior. We are indebted to him for discussing this problem with us, thereby stimulating our interest in the topics considered here, and for his constructive criticisms of our work.

It is sufficient for our purposes to require that all vector fields considered be of class $C^{2}$ at all points of the undeformed body, which points constitute a regular region of space $\Re$, as defined in (3).

1. Elasticity theory. The theory of elasticity with which we are concerned is based on the existence of a strain energy per unit of undeformed volume $\Sigma$, which is a function of displacement gradients $U^{\alpha}{ }_{\beta}$. Here $U^{\alpha}$ are the components of the displacement vector referred to a material ${ }^{1}$ coordinate $^{2}$ system and the comma denotes covariant differentiation with respect to these coordinates. We assume $\Sigma$ is of class $C^{3}$ for all $U^{\alpha}{ }_{, \beta}$, that there are no constraints on the deformation, and that inertial and body forces vanish. The basic equations may then be written

$$
\left(\partial \Sigma / \partial U_{, \beta}^{\alpha}\right)_{, \beta}=0
$$

as was shown by Kirchhoff (4).

To obtain the equations of the theory of small deformations superimposed on (possibly) large deformations, one writes $U^{\alpha}=V^{\alpha}+W^{\alpha}$, linearizes Eq. (1) with respect to the $W^{\alpha}$, and assumes that the displacement $V^{\alpha}$ satisfies (1).

Received September 13, 1955.

1The adjective "Lagrangian" is used more frequently. For reasons pointed out in $(7, \S 14)$, "material" is preferable. 
We thus have

$$
\left\{\begin{array}{l}
\partial \Sigma / \partial U^{\alpha}{ }_{, \beta}=\partial \Sigma / \partial V^{\alpha}{ }_{, \beta}+W^{\gamma}{ }_{, \delta} \partial^{2} \Sigma / \partial V^{\alpha}{ }_{, \beta} \partial V^{\gamma}{ }_{, \delta}, \\
\left(\partial \Sigma / \partial V^{\alpha}{ }_{, \beta}\right)_{, \beta}=0, \\
\left(W^{\gamma}{ }_{, \delta} \partial^{2} \Sigma / \partial V^{\alpha}{ }_{, \beta} \partial V^{\gamma}{ }_{, \delta}\right)_{, \beta}=0 .
\end{array}\right.
$$

Alternative formulations are given in (1) and $(\mathbf{6}, \S 55)$. Here $V^{\alpha}$ is regarded as known, whereas $W^{\alpha}$ is to be determined by the linear equations (2) and appropriate boundary conditions. A displacement boundary value problem is set by specifying $W^{\alpha}$ on the bounding surface $\subseteq$ of $\Re$. To show that two displacement vectors satisfying these same conditions are identical, it suffices, because of linearity, to show that any $W^{\alpha}$ which satisfies (2) and vanishes on $\subseteq$ must vanish in $\Re$. For equations (1), one type of stress boundary value problem is set by specifying $N_{\beta} \partial \Sigma / \partial U^{\alpha}{ }_{\beta}$ on $\mathfrak{S}, N_{\alpha}$ being a unit vector normal to $\Im$. In the above linearized theory, this leads to a problem in which the quantities

$$
T_{\alpha} \equiv N_{\beta} W_{, \delta}^{\gamma} \partial^{2} \Sigma / \partial V^{\alpha}{ }_{, \beta} \partial V^{\gamma}, \delta
$$

are specified on $\Im$.

If one sets $V^{\alpha}{ }_{, \beta}=0$ in (2), $W^{\alpha}$ becomes a small deformation about the state of zero deformation. It is customary to assume that $\partial \Sigma / \partial V^{\alpha}{ }_{\beta}=0$ when $V^{\alpha}{ }_{, \beta}=0$. We make no use of this assumption. If one places certain restrictions on $\partial^{2} \Sigma / \partial V^{\alpha}{ }_{, \beta} \partial V^{\gamma}{ }_{, \delta}$ evaluated at $V^{\alpha}{ }_{, \beta}=0$, one obtains the usual equations of the classical linear theory of elasticity. For example, for isotropic materials, one takes

$$
\partial^{2} \Sigma /\left.\partial V_{, \beta}^{\alpha} \partial V_{, \delta}^{\gamma}\right|_{V \rho, \sigma=0}=\lambda \delta_{\alpha}^{\beta} \delta_{\gamma}^{\delta}+\mu\left(\delta_{\gamma}^{\beta} \delta_{\alpha}^{\delta}+G^{\beta \delta} G_{\alpha \gamma}\right)
$$

where $\lambda$ and $\mu$ are the Lamé constants and $G_{\alpha \beta}$ is the metric tensor. The boundary data (3), which become

$$
T_{\alpha} \equiv \lambda W_{, \beta}^{\beta} N_{\alpha}+\mu\left(W_{\alpha, \beta}+W_{\beta, \alpha}\right) N^{\beta}
$$

when (4) holds, is the data ordinarily prescribed in stress boundary value problems in the linear theory. The Kirchhoff uniqueness proof, valid when $(3 \lambda+2 \mu) \mu>0$, establishes that $T_{\alpha}=0$ on $\subseteq$ implies that $W_{\alpha, \beta}+W_{\beta, \alpha}=0$ in $\Re$. In other words, the boundary data (5) determines the displacement field $W^{\alpha}$ to within an infinitesimal rigid motion. It seems reasonable to expect that this uniqueness theorem will hold for the small deformation when $V^{\alpha}{ }_{, \beta} \neq 0$ if suitable restrictions are placed on $\Sigma$ and $V^{\alpha}$. What constitutes a set of "suitable restrictions" on $\Sigma$ is, according to Truesdell (8), the main open problem in the theory of finite elastic deformations. We shall show that the desired uniqueness does not follow from Hadamard's stability condition.

2. Elastic stability. Hadamard $(2, \S 269)$ calls a deformation stable whenever the second variation in total strain energy is non-negative for all variations in $U^{\alpha}$ which vanish on $\mathfrak{S}$. Formally, stability means that 


$$
\Phi \equiv \delta^{2} \int_{\Re} \Sigma d V \geqslant 0
$$

whenever $\delta U^{\alpha}=\delta^{2} U^{\alpha}=0$ on $\mathfrak{S}, d V$ being the volume element. We have $\Phi=\Phi_{1}+\Phi_{2}$, where

$$
\begin{aligned}
\Phi_{1} & =\int_{\Re} \delta^{2} U^{\alpha}{ }_{, \beta} \partial \Sigma / \partial U^{\alpha}{ }_{, \beta} d V, \\
\Phi_{2} & =\int_{\Re} \delta U^{\alpha}{ }_{, \beta} \delta U^{\gamma}{ }_{, \delta} \partial^{2} \Sigma / \partial U^{\alpha}{ }_{, \beta} \partial U^{\gamma}{ }_{,} d V .
\end{aligned}
$$

From (1), (6) and (7),

$$
\Phi_{1}=\int_{\Re}\left(\delta^{2} U^{\alpha} \partial \Sigma / \partial U^{\alpha}{ }_{, \beta}\right)_{, \beta} d V=\oint_{S^{2}} \delta^{2} U^{\alpha} \partial \Sigma / \partial U^{\alpha}{ }_{, \beta} d S_{\beta}=0
$$

where $d S_{\beta}$ is the vector element of area. Similarly, from (6) and (7),

$$
\Phi_{2}=-\int_{\Re} \delta U^{\alpha}\left(\delta U^{\gamma}{ }_{, \delta} \partial^{2} \Sigma / \partial U_{, \beta}^{\alpha} \partial U^{\gamma}{ }_{, \delta}\right)_{, \beta} d V .
$$

Thus (6) can be replaced by

$$
\Phi_{2} \geqslant 0 \text { wherever } \delta U^{\alpha}=0 \text { on } \Im,
$$

$\Phi_{2}$ being given by (7) or (8). An analysis made by Kelvin (5) suggests that it is desirable to distinguish neutral or labile stability, for which $\Phi_{2}=0$ for some $\delta U^{\alpha} \not \equiv 0$, from ordinary stability, for which $\Phi_{2}=0$ implies $\delta U^{\alpha} \equiv 0$, and we find it essential for our purposes to make this distinction. Henceforth, "stability" means ordinary stability, neutral stability being excluded. There was no reason for Hadamard to make this distinction since the results which he obtained are insensitive to it.

3. Uniqueness. We begin by proving a uniqueness theorem for displacement boundary value problems.

THEOREM 1. In the theory of small deformations superimposed on large, if the large deformation is stable, the displacement boundary value problem for the small deformation has at most one solution.

Proof. Let $W^{\alpha}$ be any solution of (2) such that $W^{\alpha}=0$ on $\Im$. Multiplying the last of equations (2) by $W^{\alpha}$, summing on $\alpha$, and integrating the result over $\Re$, we obtain

$$
\int_{\Re} W^{\alpha}\left(W_{, \delta}^{\gamma} \partial^{2} \Sigma / \partial V_{, \beta}^{\alpha} \partial V_{, \delta}^{\gamma}\right)_{, \beta} d V=0 .
$$

From this and (8), we see that $\Phi_{2}$, evaluated for $U^{\alpha}=V^{\alpha}$ and $\delta U^{\alpha}=W^{\alpha}$, vanishes. If $V^{\alpha}$ is stable, $\Phi_{2}=0$ implies $W^{\alpha} \equiv 0$. Thus, if a solution exists for a given displacement boundary value problem, it is unique. 
We now proceed to determine necessary and sufficient conditions for the stability of the state of zero deformation of isotropic materials. In this case (4) holds and we obtain from (7) with $U^{\alpha}{ }_{, \beta}=0$,

$$
\Phi_{2}=\int_{\Re}\left[\lambda\left(\delta U^{\alpha}{ }_{, \alpha}\right)^{2}+\mu\left(\delta U^{\alpha}{ }_{, \beta} \delta U^{\beta}{ }_{, \alpha}+\delta U^{\alpha, \beta} \delta U_{\alpha, \beta}\right)\right] d V .
$$

Using the fact that $\delta U^{\alpha}=0$ on $\mathfrak{\subseteq}$, we have

$$
\begin{aligned}
0 & =\int_{S^{S}}\left[\delta U^{\alpha} \delta U^{\beta}{ }_{, \beta}-\delta U^{\beta} \delta U^{\alpha}{ }_{, \beta}\right] d S_{\alpha}=\int_{\Re}\left[\delta U^{\alpha} \delta U^{\beta}{ }_{, \beta}-\delta U^{\beta} \delta U^{\alpha}{ }_{, \beta}\right]_{, \alpha} d V \\
& =\oint_{\left.\Re^{[}\left[\delta U^{\alpha}{ }_{, \alpha}\right)^{2}-\delta U^{\beta}{ }_{, \alpha} \delta U^{\alpha}{ }_{, \beta}\right] d V .}
\end{aligned}
$$

Also,

$$
\delta U^{\alpha, \beta} \delta U_{\alpha, \beta}=\delta U^{\alpha}{ }_{, \beta} \delta U^{\beta}{ }_{, \alpha}+2 \omega^{\alpha \beta} \omega_{\alpha \beta},
$$

where $2 \omega_{\alpha, \beta}=U_{\alpha, \beta}-U_{\beta, \alpha}$. Using these relations, we obtain

$$
\Phi_{2}=(\lambda+2 \mu) \int_{\Re}\left(\delta U^{\alpha}{ }_{, \alpha}\right)^{2} d V+2 \mu \int_{\Re} \omega^{\alpha \beta} \omega_{\alpha \beta} d V,
$$

a result due to Kelvin (5). Since each integral is non-negative, we have stability, or at least neutral stability, of zero deformation, so long as $\lambda+2 \mu \geqslant 0$ and $\mu \geqslant 0$. A slightly sharper result is easily obtained.

Lemma. For stability of the state of zero deformation of an isotropic elastic material, it is necessary and sufficient that $\lambda+2 \mu>0$ and $\mu>0$.

Proof of sufficiency. If $\lambda+2 \mu>0$ and $\mu>0$, it is clear from (9) that $\Phi_{2} \geqslant 0$, the equality holding if and only if $\delta U^{\alpha}{ }_{, \alpha}=\omega^{\alpha}{ }_{\beta}=0$. These conditions imply that $\delta U_{\alpha}=\phi_{, \alpha}$, where $\phi$ is harmonic. Since $\phi_{, \alpha}=0$ on $\mathfrak{S}$ and $\phi$ is harmonic in $\Re, \phi_{, \alpha} \equiv 0$. Hence $\Phi_{2}>0$, unless $\delta U^{\alpha} \equiv 0$.

Proof of necessity. To show that $\Phi_{2}>0$ implies $\lambda+2 \mu>0$, it suffices to construct functions $\delta U^{\alpha}$ such that $\delta U^{\alpha}=0$ on $\mathfrak{S}, \omega^{\alpha}{ }_{\beta} \equiv 0$ in $\Re, \delta U^{\alpha}{ }_{, \alpha} \not \equiv 0$, as is clear from (9). One can take $\delta U_{\alpha}=\psi_{, \alpha}$, where $\psi$ is any function, not a constant, whose gradient vanishes on $\mathfrak{S}$. For example if, $\subseteq \subset \Re$ is a sphere of radius $r_{0}>0$, we may take $\psi=0$ in $\Re-\left(\mathfrak{S}, \psi=\left(r-r_{0}\right)^{4}\right.$ in $\mathfrak{E}$, where $r$ denotes the distance measured from the center of $\mathfrak{E}$. Similarly, to show that $\Phi_{2}>0$ implies $\mu>0$, one need only construct $\delta U^{\alpha}$ with $\delta U^{\alpha}=0$ on $\mathfrak{S}$, $\delta U^{\alpha}{ }_{, \alpha} \equiv 0, \delta U^{\alpha} \not \equiv 0$, and use (9). Such variations are easily constructed.

Theorem 2. For the stress boundary value problem (3), stability of the deformation $V^{\alpha}$ does not imply that the displacements $W^{\alpha}$ will be determined to within an infinitesimal rigid motion.

Proof. It suffices to establish that uniqueness does not follow from stability in the special case when $V^{\alpha}{ }_{, \beta}=0$ and (4) holds. By the lemma, we have stability if $3 \lambda+2 \mu=0, \mu>0$. It follows, using (4), that (2) is satisfied by any $W^{\alpha}$ such that $W^{\alpha}{ }_{, \beta}=a \delta^{\alpha}{ }_{\beta}$, where $a$ is an arbitrary constant. For any 
such displacement, (5), with $3 \lambda+2 \mu=0$, gives $T_{\alpha}=0$ for arbitrary $N_{\alpha}$. If $a \neq 0$, the displacement considered above is not an infinitesimal rigid motion, whence the theorem follows.

This theorem indicates that the definition of stability used here leads to results in disagreement with the intuitive notion, expounded by many writers in stability, that such non-uniqueness should be associated with instability. This might be regarded as an indication that it would be desirable to introduce further criteria to enable one to refine further the classification of types of stability used here.

THEOREM 3. Neutral stability of the deformation $V^{\alpha}$ does not imply uniqueness of solutions to displacement boundary value problems in the theory of small deformations superimposed on large.

Proof. Again it suffices to establish the theorem in the special case when (4) holds with $\lambda+2 \mu=0, \mu>0$. From (9) and the lemma, we then have neutral stability, but not stability. From the proof of the lemma, we can construct functions $W^{\alpha}=\psi^{, \alpha}$ such that $W^{\alpha}=0$ on $\subseteq, W^{\alpha} \not \equiv 0$ in $\Re$. It follows easily, using (4), that when $\lambda+2 \mu=0$, any such displacement satisfies (2). Since $W^{\alpha} \equiv 0$ is another solution satisfying the same boundary conditions, we do not have uniqueness.

Theorems 1 and 3 illustrate the importance of distinguishing between ordinary and neutral stability. As is pointed out by Whittaker (9, pp. 145148), the case $\lambda+2 \mu=0, \mu>0$ is of some historical interest, having been considered as an aether theory.

\section{REFERENCES}

1. A. E. Green, R. S. Rivlin, and R. T. Shield, General theory of small elastic deformations superposed on finite elastic deformations, Proc. Roy. Soc. London (A), 211 (1951), 128-154.

2. J. Hadamard, Legons sur la propagation des ondes et les équations de l'hydrodynamique (Paris, 1903).

3. O. D. Kellogg, Foundations of Potential Theory (New York, 1929).

4. G. Kirchhoff, Ueber die Gleichungen des Gleichgewichts eines elastischen Körpers bei nicht unendlich kleinen Verschiebungen seiner Theile, Akad. Wiss. Wien Sitz., 9 (1852), 762773.

5. W. Thomson (Lord Kelvin), On the reflexion and refraction of light, Phil. Mag., 26 (1888), $414-425$.

6. C. Truesdell, The mechanical foundations of elasticity and fluid dynamics, J. Rational Mech. Anal., 1 (1952), 125-300.

7. C. Truesdell, The Kinematics of Vorticity (Bloomington, 1954).

8. C. Truesdell, Das ungelöste Hauptproblem der endlichen Elastizitätstheorie, to appear in Z.a.M.M.

9. E. Whittaker, A History of the Theories of Aether and Electricity (New York, 1951).

Applied Mathematics Branch, Naval Research Laboratory, Washington, D.C. 